\title{
One \& done: treating cord compression with single-fraction radiation therapy
}

\author{
Divya Yerramilli ${ }^{1}$, Charles B. Simone $\mathrm{II}^{2}$, Kavita V. Dharmarajan ${ }^{3}$ \\ ${ }^{1}$ Department of Radiation Oncology, Massachusetts General Hospital, Boston, MA, USA; ${ }^{2}$ Department of Radiation Oncology, New York Proton \\ Center, New York, NY, USA; ${ }^{3}$ Department of Radiation Oncology and Brookdale, Department of Geriatrics and Palliative Medicine, Icahn School \\ of Medicine at Mount Sinai, New York, NY, USA \\ Correspondence to: Kavita V. Dharmarajan, MD, MSc. Department of Radiation Oncology, Brookdale Department of Geriatrics and Palliative \\ Medicine, Mount Sinai Hospital, Icahn School of Medicine at Mount Sinai, New York, NY, USA. Email: kavita.dharmarajan@mountsinai.org. \\ Provenance: This is an invited Editorial article commissioned by the Editors-in-Chief, Charles B. Simone II (New York Proton Center, New York, \\ NY, USA). \\ Comment on: Donovan EK, Sienna J, Mitera G, et al. Single versus multifraction radiotherapy for spinal cord compression: A systematic review and \\ meta-analysis. Radiother Oncol 2019;134:55-66.
}

Submitted Jul 07, 2019. Accepted for publication Jul 11, 2019.

doi: 10.21037/apm.2019.07.05

View this article at: http://dx.doi.org/10.21037/apm.2019.07.05

For patients with advanced cancer diagnosed with metastatic epidural spinal cord compression (MESCC), radiation therapy can be an appropriate and effective strategy to preserve neurologic function and improve pain (1-3). However, these patients may also have poor performance status and/ or limited prognoses (4), and multiple daily treatments using standard fractionation radiation therapy may impose substantial treatment burdens for patients and their caregivers. While several recent articles in Annals of Palliative Medicine have detailed the more widespread use of and data supporting single fraction radiotherapy for uncomplicated bone metastases (5-7), in conventional practice radiation therapy for MESCC is often administered over 2 weeks, most often to $30 \mathrm{~Gy}$ in 10 fractions. This has been justified by the delivery of a higher biologically equivalent dose (BED) with the intent to achieve more durable local control for a condition where local control can be the difference between effective treatment and tumor progression causing spinal cord compromise and paralysis $(2,8)$. More recently, studies have shown equivalent outcomes with approximately a week (20 Gy in 5 fractions) of treatment $(1,3)$.

For some patients, however, even 1 week of treatment may be onerous. These patients may have severely compromised function and/or pain at baseline. Daily transportation to radiation therapy, positioning on the treatment couch, and immobilization may all acutely worsen discomfort. There is a growing impetus to reduce the amount of treatments wherever possible without compromising clinical outcomes, to maximize the benefit for a patient, to minimize treatmentrelated burdens on patients and families, and to utilize healthcare resources for the delivery of optimal care in a responsible, cost-conscious manner.

While several retrospective studies have found similar rates of ambulatory ability and motor function preservation $(1,8)$, there has been significant reluctance to use singlefraction radiation therapy (SFRT) in lieu of multi-fraction radiation therapy (MFRT) specifically for the treatment of MESCC. This largely stems from concerns that SFRT may not preserve motor function as well as MFRT.

The meta-analysis of SFRT versus MFRT for MESCC recently published in May 2019 in Radiotherapy \& Oncology by Donovan et al. sought to quantitatively assess whether patients treated with SFRT experienced similar outcomes compared to those treated with MFRT (9). The authors performed a comprehensive systematic search of the literature from 1946 to 2018 for randomized and nonrandomized studies from multiple databases, including a search for abstracts presented at American Society of Clinical Oncology (ASCO) and American Society for Radiation Oncology (ASTRO) and unpublished or ongoing trials from several cooperative groups (RTOG, NRG Oncology, and the National Institutes of Health). The analysis was restricted to prospective randomized studies with low heterogeneity, with tests for bias and sensitivity 
analysis performed. This systematic review ultimately found three prospective randomized controlled trials fitting criteria for inclusion: Maranzano et al., SCORAD-III, and ICORG 05-03 (10-12).

The meta-analysis represented pooled data from a total of 1,126 patients treated across over 50 centers internationally, of whom 712 were available for the systematic review. The primary diagnoses were breast, prostate, and lung cancers, and a proportion of non-specified "others". All subjects were deemed non-operative candidates. Importantly, the meta-analysis was designed to examine functional outcomes rather than radiographic local control, and for this reason it consistently defined motor function on the Tomita scale or ambulatory status, and urinary continence on physician reported outcomes or need for catheterization $(9,13)$. The primary outcome in all of these studies was motor response, and there was no difference observed in those patients treated with SFRT versus MFRT. It appeared that there was mildly worse bladder dysfunction in patients treated with SFRT, although the authors acknowledged this was largely observed from low-quality evidence. Importantly, there was no difference in pain response. Pain, however, was only measured in 2 of the 3 studies, with incomparable pain scales. Quality of life (QOL), which was measured in two studies with EORTC QLQ 30, strongly favored SFRT. Toxicity reporting was variable but in SCORAD III, were no differences in grade 3-4 toxicities between SFRT and MFRT groups. Taken together, these findings support the safety and non-inferiority of single-fraction radiation therapy for MESCC, and they argue for SFRT as an effective approach to the treatment of MESCC patients with limited prognosis.

While this was the first modern meta-analysis on the topic and a commendable report, there were some limitations to this study. First, a limited number of patients and studies were included in the analysis, and those studies included heterogenous endpoints and patient populations. Additionally, although MESCC may most optimally be managed with initial surgery followed by radiation therapy, the trials included were limited to patients who were inoperable candidates. Operability in MESCC patients is determined by a myriad of factors, including a prognosis of at least 3 months, reasonable performance status, and anticipation that the benefits will outweigh the risks, which can often be a highly subjective determination. Inherent to this determination is an estimation of prognosis and life expectancy. All three trials included patients with either limited prognosis, reasonable prognosis, or no specification at all $(10-12,14)$, but overall survival in all three studies was approximately 4 months with no differences between treatment arms. Regardless of the variability in prognostic estimates of included patients in the studies comprising this meta-analysis, it is known that physicians often dramatically over-estimate prognosis $(13,15,16)$. In the report by Christakis et al. from nearly two decades ago, still considered to be a seminal paper on prognostication, physician estimates of survival overestimated actual survival by a factor of 5.3 (17).

This may be the most challenging hurdle for appropriately selecting patients for SFRT in the setting of MESCC. Radiation oncologists can consider using available prognostic tools to help limit the variability in prognostic estimation, such as the TEACHH model or the scoring system reported on by Rades et al. (16,18-21). With limited prognoses of less than 3 months, which largely reflects the population included in Donovan's meta-analysis, clinicians should be confident in choosing SFRT, particularly since MFRT may not only be resource-intensive, but timeintensive in a patient who does not have much time remaining. In patients estimated to have a prognosis of greater than 6 months, there may be other factors that support treatment with higher BEDs, such as MFRT or stereotactic body radiation therapy (SBRT) for maximal local control, pain response, and preservation of function. Rades et al. retrospectively reported higher local failure rates from 5-fraction MFRT and SFRT compared with 1020 fraction regimens at up to 12 months after RT, further underscoring the importance of higher BED radiotherapy regimens at more extended time intervals (22). Thus, the decision to offer SFRT hinges on the complex challenge of estimating prognosis.

Minimizing number of treatment fractions in palliative radiation also is integral to the delivery of value-based healthcare. ASTRO's Choosing Wisely campaign has advocated for treating patients with bone metastases in no more than 10 fractions, and the American Academy of Hospice and Palliative Medicine (AAHPM) has further recommended the use of a single fraction for uncomplicated bone metastases $(23,24)$. Single-fraction radiation therapy can additionally offer many benefits, such as improved access to treatment; judicious use of healthcare resources; limited systemic treatment breaks; and reduced pain and inconvenience of daily transportation, set-up, immobilization, and treatment. Despite these guidelines, patterns in the United States have shown that physicians favor multi-fraction therapy for palliative 
radiation, with ten-fraction treatment still the most commonly employed, followed by five-fraction treatment. There are several hypotheses as to why physicians favor multi-fraction treatment for palliative intent radiation therapy (25). First, MFRT is likely the preferred treatment approach for patients thought to have a more prolonged life expectancy, and, as previously mentioned, physicians often overestimate patient prognosis. Second, conventional practice habits and historical evidence supports the use of standard fractionation therapy, and physicians may have more control over anticipating and managing side effects with this approach. Additionally, in the United States, the current correlation between reimbursement and the number of fractions delivered unfortunately can pose as a barrier to the widespread adoption of SFRT when clinically appropriate (26). This is seen less in countries that have nationalized healthcare (27). If these practice patterns do not change now, future generations of radiation oncologists will continue to train under a paradigm that prefers multifraction treatment, and they may be reluctant to use fractionation schemes with which they are unfamiliar.

While all of these ethical arguments in conjunction with the evidence from Donovan et al.'s meta-analysis support SFRT, several questions persist. The meta-analysis did not include patients with prior surgery or prior radiation and did not specify histologies beyond breast, lung, prostate or those that were otherwise designated as favorable such as seminoma and small cell lung. Thus, it is difficult to draw conclusions on the efficacy of SFRT in patients with radioresistant histologies such as colorectal cancer or renal cell carcinoma. It is also unclear as to the rates and settings in which re-treatments were indicated and offered. Patients living with controlled systemic disease will undoubtedly also be an area for further investigation. In these settings, the role of multi-fraction therapy may be inevitable. In patients with a more extended life expectancy or for whom durable local control or dose-escalation may otherwise be desired, MFRT or even SBRT can still play an important and optimal role.

In the earliest descriptions of palliative radiation therapy, Robert Parker argued in 1964 that, "When the initial objective of radiation therapy is palliation, new ground rules must be applied. Possible serious complications or even slowly self-limiting side effects of treatment are no longer acceptable. Overall treatment time must be short. Cost must be minimized. Convenience of treatment must be considered." (28). This philosophy lies at the center of Donovan et al.'s metaanalysis, which presents compelling data to support the use of the shortest treatment course whenever possible, as long as it is clinically meaningful. In the patient populations studied, Donovan et al. has shown clinical equipoise between SFRT and MFRT in terms of motor response, maintenance of ambulatory status, pain, overall survival, and toxicity, and superior of SFRT in terms of QOL, despite some limitations inherent to the patient population examined. Finally, while this meta-analysis of pooled, randomized data is compelling, the paucity of trials across various clinical settings in MESCC should serve as a broader call to action for more trial development in palliative oncology. Radiation oncologists must continue to lead high quality research efforts to optimize local treatment of metastatic disease in a manner that places a high priority on improving symptoms and quality of life outcomes which are directly relevant to patients.

\section{Acknowledgments}

None.

\section{Footnote}

Conflicts of Interest: The authors have no conflicts of interest to declare.

Ethical Statement: The authors are accountable for all aspects of the work in ensuring that questions related to the accuracy or integrity of any part of the work are appropriately investigated and resolved.

\section{References}

1. Rades D, Stalpers LJ, Veninga T, et al. Evaluation of five radiation schedules and prognostic factors for metastatic spinal cord compression. J Clin Oncol 2005;23:3366-75.

2. Rades D, Panzner A, Rudat V, et al. Dose escalation of radiotherapy for metastatic spinal cord compression (MSCC) in patients with relatively favorable survival prognosis. Strahlenther Onkol 2011;187:729-35.

3. Rades D, Šegedin B, Conde-Moreno AJ, et al. Radiotherapy with $4 \mathrm{~Gy} \times 5$ versus $3 \mathrm{~Gy} \times 10$ for metastatic epidural spinal cord compression: final results of the SCORE-2 trial (ARO 2009/01). J Clin Oncol 2016;34:597-602.

4. Aoude A, Amiot LP. A comparison of the modified Tokuhashi and Tomita scores in determining prognosis for patients afflicted with spinal metastasis. Can J Surg 2014;57:188. 
5. Tsang DS, Yau V, Raziee H, et al. Debate: Singlefraction treatment should be standard in the retreatment of uncomplicated bone metastases. Ann Palliat Med 2015;4:207-13.

6. Chow R, Hoskin P, Hollenberg D, et al. Efficacy of single fraction conventional radiation therapy for painful uncomplicated bone metastases: a systematic review and meta-analysis. Ann Palliat Med 2017;6:125-42.

7. Malek L, Chow E, Simone CB 2nd. Speedy response can be achieved from palliative radiotherapy in the treatment of painful uncomplicated bone metastases. Ann Palliat Med 2017;6:S120-1.

8. Rades D, Huttenlocher S, Šegedin B, et al. Single-fraction versus 5 -fraction radiation therapy for metastatic epidural spinal cord compression in patients with limited survival prognoses: results of a matched-pair analysis. Int J Radiat Oncol Biol Phys 2015;93:368-72.

9. Donovan EK, Sienna J, Mitera G, et al. Single versus multifraction radiotherapy for spinal cord compression: A systematic review and meta-analysis. Radiother Oncol 2019;134:55-66.

10. Maranzano E, Trippa F, Casale M, et al. 8 Gy singledose radiotherapy is effective in metastatic spinal cord compression: results of a phase III randomized multicentre Italian trial. Radiother Oncol 2009;93:174-9.

11. Hoskin P, Misra V, Hopkins K, et al. SCORAD III: Randomized noninferiority phase III trial of singledose radiotherapy (RT) compared to multifraction RT in patients (pts) with metastatic spinal canal compression (SCC). J Clin Oncol 2017;35:LBA10004.

12. Thirion P, O'Sullivan L, Clayton-Lea A, et al. ICORG 0503: Prospective randomized non-inferiority phase 3 trial comparing two radiation schedules in malignant spinal cord compression not proceeding with surgical decompression. Int J Radiat Oncol Biol Phys 2014;90:1263-4.

13. Tokuhashi $Y$, Uei H, Oshima M, et al. Scoring system for prediction of metastatic spine tumor prognosis. World $\mathrm{J}$ Orthop 2014;5:262.

14. Fairchild A, Debenham B, Danielson B, et al. Comparative multidisciplinary prediction of survival in patients with advanced cancer. Support Care Cancer 2014;22:611-7.

15. Glare P, Virik K, Jones M, et al. A systematic review of physicians' survival predictions in terminally ill cancer patients. Bmj 2003;327:195.

16. Rades D, Dunst J, Schild SE. The first score predicting overall survival in patients with metastatic spinal cord compression. Cancer 2008;112:157-61.

17. Christakis NA, Lamont EB. Extent and determinants of error in doctors' prognoses in terminally ill patients: prospective cohort study. BMJ 2000;320:469-72.

18. Krishnan MS, Epstein-Peterson Z, Chen Y, et al. Predicting life expectancy in patients with metastatic cancer receiving palliative radiotherapy: the TEACHH model. Cancer 2014;120:134-41.

19. Chow E, Abdolell M, Panzarella T, et al. Predictive model for survival in patients with advanced cancer. J Clin Oncol 2008;26:5863-9.

20. van der Linden YM, Dijkstra SP, Vonk EJ, et al. Prediction of survival in patients with metastases in the spinal column: results based on a randomized trial of radiotherapy. Cancer 2005;103:320-8.

21. Zeng L, Zhang L, Culleton S, et al. Edmonton symptom assessment scale as a prognosticative indicator in patients with advanced cancer. J Palliat Med 2011;14:337-42.

22. Rades D, Lange $M$, Veninga $T$, et al. Final results of a prospective study comparing the local control of shortcourse and long-course radiotherapy for metastatic spinal cord compression. Int J Radiat Oncol Biol Phys 2011;79:524-30.

23. Hahn C, Kavanagh B, Bhatnagar A, et al. Choosing wisely: the American Society for Radiation Oncology's top 5 list. Pract Radiat Oncol 2014;4:349-55.

24. Fischberg D, Bull J, Casarett D, et al. Five things physicians and patients should question in hospice and palliative medicine. J Pain Symptom Manage 2013;45:595-605.

25. Spratt DE, Mancini BR, Hayman JA, et al. Contemporary statewide practice pattern assessment of the palliative treatment of bone metastasis. Int J Radiat Oncol Biol Phys 2018;101:462-7.

26. Konski A, Yu JB, Freedman G, et al. Radiation oncology practice: adjusting to a new reimbursement model. J Oncol Pract 2016;12:e576-83.

27. Chow E, Danjoux C, Wong R, et al. Palliation of bone metastases: a survey of patterns of practice among Canadian radiation oncologists. Radiother Oncol 2000;56:305-14.

28. Parker RG. Palliative radiation therapy. JAMA 1964;190:1000-2.

Cite this article as: Yerramilli D, Simone CB 2nd, Dharmarajan KV. One \& done: treating cord compression with single-fraction radiation therapy. Ann Palliat Med 2019;8(4):356-359. doi: 10.21037/apm.2019.07.05 